\title{
Algebraic elimination of $\varepsilon$-transitions
}

\author{
Gérard H. E. Duchamp ${ }^{1}$, Hatem Hadj Kacem ${ }^{2}$ and Éric Laugerotte ${ }^{2}$ \\ ${ }^{1}$ LIPN, UMR CNRS 7030. Institut Galilée - Université Paris-Nord \\ 99, avenue Jean-Baptiste Clément 93430 Villetaneuse, France. \\ enail: gerard.dudhampalipn.univ-paris13. fr \\ ${ }^{2}$ LIFAR, Faculté des Sciences et des Techniques, 76821 Mont-Saint-Aignan Cedex, France. \\ email: \{hatem.hadj-kacem, eric.laugerotte \}aniv-raun.fr
}

received Sep 15, 2004, revised Dec 7, 2004, Feb 20, Mar 6, Apr 23, 2005, accepted Apr 26, 2005.

We here describe a method of removing the $\varepsilon$-transitions of a weighted automaton. The existence of a solution for this removal depends on the existence of the star of a single matrix which, in turn, is based on the computation of the stars of scalars in the ground semiring. We discuss two aspects of the star problem (by infinite sums and by equations) and give an algorithm to suppress the $\varepsilon$-transitions and preserve the behaviour. Running complexities are computed.

Keywords: Automata with multiplicities, $\varepsilon$-transitions, behaviour, star of matrices.

\section{Introduction}

Automata with multiplicities (or weighted automata) are a versatile class of transition systems which can modelize as well classical (boolean), stochastic, transducer automata and be applied to various purposes such as image compression, speech recognition, formal linguistic (and automatic treatment of natural languages too) and probabilistic modelling. For generalities over automata with multiplicities see [1] and [10], problems over identities and decidability results on these objects can be found in [11], [12] and [13].

A particular type of these automata are the automata with $\varepsilon$-transitions denoted by $k$ - $\varepsilon$-automata which are the result, for example, of the application of Thompson method to transform a weighted regular expression into a weighted automaton [14].

The aim of this paper is to study the equivalence between $k$ - $\varepsilon$-automata and $k$-automata. Indeed, we will present here an algebraic method in order to compute, for a weighted automaton with $\varepsilon$-transitions (choosen in a suited class) an equivalent weighted automaton without $\varepsilon$-transitions which has the same behaviour. Here, the closure of $\varepsilon$-transitions implies the existence of the star of transition matrix for $\varepsilon$. Its running time complexity is deduced from that of the matrix multiplication in $k^{n \times n}$. In the case of wellknown semirings (like boolean and tropical), the closure is computed in $\mathrm{O}\left(n^{3}\right)$ [15]. We fit the running time complexity to the case when $k$ is a ring.

The structure of the paper is the following. We first recall (in Section 2) the notions of a semiring and the computation of the star of matrices. 
After introducing (in Section 3 ) the notions of a $k$-automaton and $k$ - $\varepsilon$-automaton, we present (in Section 4 and 5 ) our principal result which is a method of elimination of $\varepsilon$-transitions and show particular cases of series on which our result can be applied. In Section 6 , we give the equivalence between the two types of automata and discuss its validity. A conclusion section ends the paper.

\section{Acknowledgements}

We thank the anonymous referees for careful reading and fruitful remarks.

\section{Semirings}

In the following, a semiring $\left(k, \oplus, \otimes, 0_{k}, 1_{k}\right)$ is a set together with two laws and their neutrals. More precisely $\left(k, \oplus, 0_{k}\right)$ is a commutative monoid with $0_{k}$ as neutral and $\left(k, \otimes, 1_{k}\right)$ is a monoid with $1_{k}$ as neutral. The product is distributive with respect to the addition and zero is an annihilator $\left(0_{k} \otimes x=\right.$ $\left.x \otimes 0_{k}=0_{k}\right)$ [7]. For example all rings are semirings, whereas $(\mathbb{N},+, \times, 0,1)$, the boolean semiring $\mathbb{B}=$ $(\{0,1\}, \vee, \wedge, 0,1)$ and the tropical semiring $\mathbb{T}=\left(\mathbb{R}_{+} \cup\{\infty\}\right.$, min, $\left.+, \infty, 0\right)$ are well-known examples of semirings that are not rings. The star of a scalar is introduced by the following definition:

Definition 1 Let $x \in k$, the scalar $y$ is a right (resp. left) star of $x$ if and only if $(x \otimes y) \oplus 1_{k}=y$ (resp. $\left.(y \otimes x) \oplus 1_{k}=y\right)$.

If $y \in k$ is a left and right star of $x \in k$, we say that $y$ is a star for $x$ and we write $y=x^{\circledast}$.

Remark 1 Left or right stars need not exist and need not coincide (see examples below).

\section{$\operatorname{Example(s)~} 1$}

(1) For $k=\mathbb{C}$, any complex number $x \neq 1$ has a unique star which is $y=(1-x)^{-1}$. In the case $|x|<1$, we observe easily that $y=1+x+x^{2}+\cdots$.

(2) Let $k$ be the ring of all linear operators $(\mathbb{R}[x] \rightarrow \mathbb{R}[x])$. Let $X$ and $Y_{\alpha}$ defined by $X\left(x^{0}\right)=1$, $X\left(x^{n}\right)=x^{n}-n x^{n-1}$ with $n>0$ and $Y_{\alpha}\left(x^{n}\right)=(n+1)^{-1} x^{n+1}+\alpha$ with $\alpha \in \mathbb{R}$. Then $X Y_{\alpha}+1=Y_{\alpha}$ and an infinite number of solutions exist for the right star (which is not a left star if $\alpha \neq 0$ ).

(3) For $k=\mathbb{T}$ (tropical semiring), any number $x>0$ has a unique star $y=1$.

We can observe that if the opposite $-x$ of $x$ exists then right (resp. left) stars of $x$ are right (resp. left) inverses of $(1 \oplus(-x))$ and conversely. Thus, if they exist, any right star $x^{\circledast_{r}}$ equals any left star $x^{\circledast l}$ as $x^{\circledast_{l}}=x^{\circledast_{l}} \otimes\left((1 \oplus(-x)) \otimes x^{\circledast_{r}}\right)=\left(x^{\circledast_{l}} \otimes(1 \oplus(-x))\right) \otimes x^{\circledast_{r}}=x^{\circledast_{r}}$. In this case, the star is unique.

If $Q$ is a finite set, the space $k^{Q \times Q}$ of square matrices with indices in $Q$ and coefficients in $k$ has a natural semiring structure with the usual operations (sum and product). A (right) star of $M \in k^{Q \times Q}$ (when it exists) is a solution of the equation $M Y+1_{Q \times Q}=Y$ (where $1_{Q \times Q}$ is the identity matrix). Let $M \in k^{Q \times Q}$ be given by

$$
M=\left(\begin{array}{ll}
a_{11} & a_{12} \\
a_{21} & a_{22}
\end{array}\right)
$$


where $a_{11} \in k^{Q_{1} \times Q_{1}}, a_{12} \in k^{Q_{1} \times Q_{2}}, a_{21} \in k^{Q_{2} \times Q_{1}}$ and $a_{22} \in k^{Q_{2} \times Q_{2}}$ such that $Q_{1}+Q_{2}=Q$. Let $N \in k^{Q \times Q}$ given by

$$
N=\left(\begin{array}{ll}
A_{11} & A_{12} \\
A_{21} & A_{22}
\end{array}\right)
$$

with

$$
\begin{aligned}
& A_{11}=\left(a_{11}+a_{12} a_{22}{ }^{*} a_{21}\right)^{*} \\
& A_{12}=a_{11}{ }^{*} a_{12} A_{22} \\
& A_{21}=a_{22}{ }^{*} a_{21} A_{11} \\
& A_{22}=\left(a_{22}+a_{21} a_{11}{ }^{*} a_{12}\right)^{*}
\end{aligned}
$$

Theorem 1 If the right hand sides of formulas (1), (2), (3) and (4) are defined, the matrix $M$ admits $N$ as a right star.

Proof. Suppose, without loss of generality, that $Q=[1, n]_{\mathbb{N}} ; n=p+q ; Q_{1}=[1, p]_{\mathbb{N}} ; Q_{2}=$ $[p+1, p+q]_{\mathbb{N}}$. We have to show that $N$ is a solution of the equation $M y+1_{n \times n}=y$. By computation, one has

$$
\begin{aligned}
M N+1 & =\left(\begin{array}{ll}
a_{11} & a_{12} \\
a_{21} & a_{22}
\end{array}\right)\left(\begin{array}{ll}
A_{11} & A_{12} \\
A_{21} & A_{22}
\end{array}\right)+\left(\begin{array}{ll}
1_{p \times p} & 0_{p \times q} \\
0_{q \times p} & 1_{q \times q}
\end{array}\right) \\
& =\left(\begin{array}{ll}
a_{11} A_{11}+a_{12} A_{21}+1_{p \times p} & a_{11} A_{12}+a_{12} A_{22} \\
a_{21} A_{11}+a_{22} A_{21} & a_{21} A_{12}+a_{22} A_{22}+1_{q \times q}
\end{array}\right)
\end{aligned}
$$

where $0_{p \times q}$ is the zero matrix in $k^{p \times q}$. We verify the relations (1), (2), (3) and (4) by:

$$
\begin{aligned}
a_{11} A_{11}+a_{12} A_{21}+1_{p \times p} & =a_{11} A_{11}+a_{12} a_{22}^{*} a_{21} A_{11}+1_{p \times p}= \\
A_{11}\left(a_{11}+a_{12} a_{22}{ }^{*} a_{21}\right)+1_{p \times p} & =A_{11} \\
a_{11} A_{12}+a_{12} A_{22} & =a_{11} a_{11}{ }^{*} a_{12} A_{22}+a_{12} A_{22}= \\
\left(a_{11} a_{11}{ }^{*}+1\right) a_{12} A_{22} & =a_{11}{ }^{*} a_{12} A_{22}=A_{12} \\
a_{21} A_{11}+a_{22} A_{21} & =a_{21} A_{11}+a_{22} a_{22}{ }^{*} a_{21} A_{11}= \\
\left(1+a_{22} a_{22}{ }^{*}\right) a_{21} A_{11} & =a_{22}{ }^{*} a_{21} A_{11}=A_{21} \\
& \\
a_{21} A_{12}+a_{22} A_{22}+1_{q \times q} & =a_{21} a_{11}{ }^{*} a_{12} A_{22}+a_{22} A_{22}+1_{q \times q}= \\
\left(a_{22} a_{21} a_{11}{ }^{*} a 12\right) A_{22}+1_{q \times q} & =A_{22}
\end{aligned}
$$




\section{Remark 2}

(i) In [8] and [16], similar formulas are expressed for the computation of the inverse of matrices when $k$ is a division ring (this can be extended to the case of rings).

It must be emphasized that the converse of Theorem (1), of course, does not hold as shows the example below (the coefficients are taken in a ring and here the star is unique).

$$
M=\left(\begin{array}{cc}
1 & -1 \\
1 & -1
\end{array}\right) \quad M^{*}=\left(\begin{array}{cc}
2 & -1 \\
1 & 0
\end{array}\right)
$$

However, if the formulas are defined at each step of the computation (see below for a formalization of this), it provides a recursive way to compute a star of a matrix.

(ii) Similar formulas can be stated in the case of a left star. The matrix $N$ is the left star of $M$ with

$$
\begin{aligned}
& A_{11}=\left(a_{11}+a_{12} a_{22}{ }^{*} a_{21}\right)^{*} \\
& A_{12}=A_{11} a_{12} a_{22}{ }^{*} \\
& A_{21}=A_{22} a_{21} a_{11}{ }^{*} \\
& A_{22}=\left(a_{22}+a_{21} a_{11}{ }^{*} a_{12}\right)^{*}
\end{aligned}
$$

(iii) When all their terms are defined, formulas above (as well as (1), (2), (3) and (4)) are valid with matrices of any size with any block partitionning. Matrices of even size are often, in practice, partitionned into square blocks but, for matrices with odd dimensions, the approach called dynamic peeling is applied. More specifically, let $M \in k^{n \times n}$ a matrix given by

$$
M=\left(\begin{array}{ll}
a_{11} & a_{12} \\
a_{21} & a_{22}
\end{array}\right)
$$

where $n \in 2 \mathbb{N}+1$. The dynamic peeling [9] consists of cutting out the matrix in the following way: $a_{11}$ is $a(n-1) \times(n-1)$ matrix, $a_{12}$ is a $(n-1) \times 1$ matrix, $a_{21}$ is a $1 \times(n-1)$ matrix and $a_{22}$ is a scalar.

If desired, formulation of Theorem (1) can be seen as recursive in essence. In this respect, it implies that stars of submatrices could be already computed by the same scheme. This type of computation will be formalized by the notion of admissible tree of computation which we describe below.

Let $Q$ be a finite set and $\mathcal{A}[Q]$ be the set of binary trees with leaves in $Q$. It can be defined by the grammar

$$
\mathcal{A}[Q]=Q+(\mathcal{A}[Q], \mathcal{A}[Q])
$$

or, if one prefers a graded version

$$
\left\{\begin{array}{l}
\mathcal{A}_{1}[Q]=Q \\
\mathcal{A}_{n}[Q]=\sum_{i+j=n}\left(\mathcal{A}_{i}[Q], \mathcal{A}_{j}[Q]\right) \text { if } n \geq 2
\end{array}\right.
$$

Now, the list of leaves of a tree $T \in \mathcal{A}_{n}[Q]$ is a word $l v(T) \in Q^{n}$ defined by

$$
\left\{\begin{array}{l}
l v(T)=T \text { if } T \in \mathcal{A}_{1}[Q] \\
\operatorname{lv}(T)=\operatorname{lv}\left(T_{1}\right) l v\left(T_{2}\right) \text { (concatenation) if } T=\left(T_{1}, T_{2}\right) .
\end{array}\right.
$$


The set of leaves of $T$ is then $\operatorname{alph}(\operatorname{lv}(T))$, where $\operatorname{alph}(w)$ is classically the alphabet of the word $w$. We will say that $T \in \mathcal{A}[Q]$ is an admissible tree of computation for $M \in k^{Q \times Q}$ if

1. the word $l v(T)$ is standard (with no repetition) and contains all the indices (i.e. $|l v(T)|=|Q|$ and $\operatorname{alph}(\operatorname{lv}(T))=Q$ )

2. if $|Q|=1$ (thus $M \in k^{Q \times Q} \simeq k$ is a scalar), $M$ admits a star in $k^{Q \times Q}$

3. - if $|Q| \geq 2$, set $T=\left(T_{1}, T_{2}\right)$ and $Q_{i}=\operatorname{lv}\left(T_{i}\right)(i=1,2)$ then $T_{1}$ is admissible for the submatrix $\left.M\right|_{Q_{1} \times Q_{1}}$ and $T_{2}$ is admissible for the submatrix $\left.M\right|_{Q_{2} \times Q_{2}}$.

- formulas above (1), (2), (3) and (4) are defined for the partitionning $Q=Q_{1}+Q_{2}$.

The conditions above assures that the recursive computation of Theorem (1) is defined at each step and, in this case, we will say that the star of $M$ is computed along the (admissible) tree of computation $T$.

Theorem 2 Let $k$ be a semiring, $M \in k^{Q \times Q}$ a square matrix and $T$ a tree of computation admissble for $M$. Then, the right star of a matrix of size $n \in \mathbb{N}$ can be computed in $\mathrm{O}\left(n^{\omega}\right)$ operations with:

- $\omega \leq 3$ if $k$ is not a ring,

- $\omega \leq 2.808$ if $k$ is a ring,

- $\omega \leq 2.376$ if $k$ is a field.

Proof. For $n=2^{m} \in \mathbb{N}$, let $T_{m}^{+}, T_{m}^{\times}$and $T_{m}^{*}$ denote the number of operations $\oplus, \otimes$ and $\circledast$ in $k$ that the addition, the multiplication and the star of matrix respectively perform with an input of size $n$. Then

$$
\begin{aligned}
& T_{0}^{*}=1 \\
& T_{m}^{*}=2 T_{m-1}^{+}+8 T_{m-1}^{\times}+4 T_{m-1}^{*}
\end{aligned}
$$

by Theorem 1. For arbitrary semiring, one has $T_{m-1}^{+}=2^{2(m-1)}$. If $k$ is a ring, using Strassen's algorithm for the matrix multiplication [19], it is known that at most $n^{\log _{2}(7)}$ operations are necessary. If $k$ is a field, using Coppersmith and Winograd's algorithm [3], it is known that at most $n^{2.376}$ operations are necessary. Suppose that $T_{m-1}^{\times}=2^{(m-1) \omega}$. The solution of the recurrence relation (7) is

$$
4^{m}+\frac{1}{2}(m+1) 4^{m}-\frac{\left(6+2^{\omega-1}\right)}{2^{\omega}-4}+\frac{8 \cdot 2^{m \omega}}{2^{\omega}-4}
$$

where the leading term is $2^{m \omega}$.

Remark 3 In some situations, any tree $T$ with $|Q|$ leaves and standard $l v(T)$ is admissible. This is the case, for example, of matrices of series without constant term (matrices of proper series [1]). 
The running time complexity for the computation of the right (resp. left) star of a matrix depends on $T_{\oplus}$, $T_{\otimes}$ and $T_{\circledast}$, but it depends also on the representation of coefficients in machine. In the case $k=\mathbb{Z}$ for example, the multiplication of two integers is computed in $\mathrm{O}(m \log (m) \log (\log (m)))$, using FFT if $m$ bits are necessary [18].

Theorem 3 With the same hypotheses as in (2) ( $M \in k^{Q \times Q}$ a square matrix and $T$ a tree of computation admissible for $M)$, the space complexity of the right star of a matrix of size $n \in \mathbb{N}$ is $\mathrm{O}\left(n^{2} \log (n)\right)$.

Proof. For $n=2^{m} \in \mathbb{N}$ and $k$ a semiring, let $E_{m}^{*}$ denote the space complexity of operation $*$ that the star of matrix perform with an input of size $n$. Then

$$
\begin{aligned}
& E_{0}^{*}=1 \\
& E_{m}^{*}=12 \cdot 2^{2 m-1}+4 E_{m-1}^{*}
\end{aligned}
$$

The solution of the recurrence relation (8) is

$$
-5 \cdot 4^{m}+(6 m+6) 4^{m}
$$

where the leading term is $m \cdot 4^{m}$.

The running of the algorithm needs the reservation of memory spaces for the resulting matrix (the star of the input matrix) and for intermediate results stored in temporary locations.

Let $k\langle\langle\Sigma\rangle\rangle$ be the set of noncommutative formal series with $\Sigma$ as alphabet (i.e. functions on the free monoid $\Sigma^{*}$ with values in $k$ ). It is a semiring equipped with + the sum and t the Cauchy product.

We denote by $\alpha(?)$ and (?) $\alpha$ the left and right external product respectively. The star (?)* of a formal series is well-defined if and only if the star of the constant term exists [10,1].

When $\Sigma$ is finite, the set $\operatorname{RAT}_{k}(\Sigma)$ is the closure of the alphabet $\Sigma$ by sums, external and Cauchy products and the star.

\section{Automata with multiplicities}

Let $\Sigma$ be a finite alphabet and $k$ be a semiring. A weighted automaton (or linear representation) of dimension $n$ on $\Sigma$ with multiplicities in $k$ is a triplet $(\lambda, \mu, \gamma)$ where:

- $\lambda \in k^{1 \times n}$ (the input vector),

- $\mu: \Sigma \rightarrow k^{n \times n}$ (the transition function),

- $\gamma \in k^{n \times 1}$ (the output vector).

Such an automaton is usually drawn as a directed valued graph (see Figure 1). A transition $(i, a, j) \in$ $\{1, \ldots, n\} \times \Sigma \times\{1, \ldots, n\}$ connects the state $i$ with the state $j$. Its weight is $\mu(a)_{i j}$. The weight of the initial (final) state $i$ is $\lambda_{i}$ (respectively $\gamma_{i}$ ). The mapping $\mu$ induces a morphism of monoids from $\Sigma^{*}$ to 


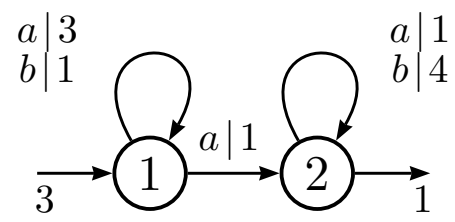

Figure 1: A $\mathbb{N}$-automaton

$k^{n \times n}$. The behaviour of the weighted automaton $\mathcal{A}$ belongs to $k\langle\langle\Sigma\rangle\rangle$. It is defined by:

$$
\operatorname{behaviour}(\mathcal{A})=\sum_{u \in \Sigma^{*}}(\lambda \mu(u) \gamma) u
$$

More precisely, the weight $\langle$ behaviour $(\mathcal{A}), u\rangle$ of the word $u$ in the formal series behaviour $(\mathcal{A})$ is the weight of $u$ for the $k$-automaton $\mathcal{A}$ (this is an accordance with the scalar product denotation $\langle S \mid u\rangle:=S(u)$ for any function $\left.S: \Sigma^{*} \rightarrow k[2]\right)$.

Example(s) 2 The behaviour of the automaton $\mathcal{A}$ of Figure 1 is

$$
\operatorname{behaviour}(\mathcal{A})=\sum_{u, v \in \Sigma^{*}} 3^{|u|_{a}+1} 4^{|v|_{b}} u a v .
$$

Let $u=a b a$. Then, its weight in $\mathcal{A}$ is:

$$
\begin{aligned}
\lambda \mu(u) \gamma & =\lambda \mu(a) \mu(b) \mu(a) \gamma \\
& =\left(\begin{array}{ll}
3 & 0
\end{array}\right)\left(\begin{array}{ll}
3 & 1 \\
0 & 1
\end{array}\right)\left(\begin{array}{ll}
1 & 0 \\
0 & 4
\end{array}\right)\left(\begin{array}{ll}
3 & 1 \\
0 & 1
\end{array}\right)\left(\begin{array}{l}
0 \\
1
\end{array}\right)=21 .
\end{aligned}
$$

The set $\operatorname{REC}_{k}(\Sigma)$ is known to be equal to the set of series which are the behaviour of a $k$-automaton. We recall the well-known result of Schützenberger [17]:

$$
\operatorname{REC}_{k}(\Sigma)=\operatorname{RAT}_{k}(\Sigma) .
$$

A $k$ - $\varepsilon$-automaton $\mathcal{A}_{\varepsilon}$ is a $k$-automaton over the alphabet $\Sigma_{\varepsilon}=\Sigma \cup \tilde{\varepsilon}$ (see Figure 2). We must keep the reader aware that $\tilde{\varepsilon}$ is considered here as a new letter and that there exists an empty word for $\Sigma_{\varepsilon}^{*}=(\Sigma \cup \tilde{\varepsilon})^{*}$ denoted here by $\varepsilon$. The transition matrix of $\tilde{\varepsilon}$ is denoted $\mu_{\tilde{\varepsilon}}$.

Example(s) 3 In Figure 2, the behaviour of the automaton $\mathcal{A}_{\varepsilon}$ is

$$
\operatorname{behaviour}\left(\mathcal{A}_{\varepsilon}\right)=18 \tilde{\varepsilon}\left(\sum_{i \in \mathbb{N}} 2^{i}(a \tilde{\varepsilon})^{i}\right) \tilde{\varepsilon}=18 \tilde{\varepsilon}(2 a \tilde{\varepsilon})^{*} \tilde{\varepsilon}
$$




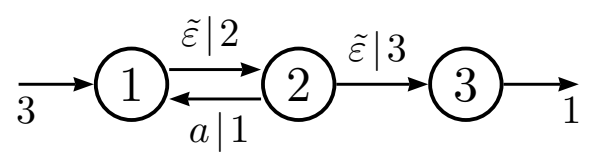

Figure 2: A $\mathbb{N}-\varepsilon$-automaton

\section{Algebraic elimination}

Let $\Phi$ be the morphism from $\Sigma_{\varepsilon}^{*}$ to $\Sigma^{*}$ induced by

$$
\left\{\begin{array}{l}
\Phi(x)=x \quad \text { if } x \in \Sigma, \\
\Phi(\tilde{\varepsilon})=\varepsilon .
\end{array}\right.
$$

It is classical that the morphism $\Phi$ can be uniquely extended to the polynomials of $k\left\langle\Sigma_{\varepsilon}\right\rangle$ as a morphism of algebras $k\left\langle\Sigma_{\varepsilon}\right\rangle \mapsto k\langle\Sigma\rangle$ by, for $P$ a polynomial,

$$
\Phi(P)=\Phi\left(\sum_{u \in \Sigma^{*}}\langle P \mid u\rangle u\right)=\sum_{u \in \Sigma^{*}}\left(\sum_{\Phi(v)=u}\langle P \mid v\rangle\right) u
$$

as, in this case, the sum

$$
\sum_{\Phi(v)=u}\langle P \mid v\rangle
$$

is finite-supported and then well defined. But, we remark that the set of preimages of $u=a_{1} a_{2} \ldots a_{n}$ by $\Phi$ is

$$
\{v \mid \Phi(v)=u\}=\tilde{\varepsilon}^{*} a_{1} \tilde{\varepsilon}^{*} a_{2} \cdots \tilde{\varepsilon}^{*} a_{n} \tilde{\varepsilon}^{*}
$$

This shows that, in this case, all preimages are infinite and we will discuss on the convergence of the sum $\sum_{\Phi(v)=u}\langle P \mid v\rangle$.

BIn the sequel, we will extend formula (9) in two ways:

1. To the series for which the sum (10) remains with finite support (this set is larger than the polynomials and includes also the behaviours of $\varepsilon$-automata with an acyclic $\epsilon$-transition matrix). We will call them $\Phi$-finite series (FF series).

2. Having supposed the semiring endowed with a topology (or, at least, an "infinite sums" function) we define the set of series for which the sums (10) converge (this definition covers the behaviour of classical boolean $\varepsilon$-automata). We will call them $\Phi$-convergent series (FC series).

After these extensions, we will prove that the behaviour of the automaton obtained by algebraic elimination is the image by $\Phi$ (the erasure of $\varepsilon$ ) of the behaviour (in $k\left\langle\left\langle\Sigma_{\varepsilon}\right\rangle\right\rangle$ ) of the initial automaton. 


\section{FF and FC series}

\subsection{FF ( $\Phi$-finite) series}

Let $S \in k\left\langle\left\langle\Sigma_{\varepsilon}\right\rangle\right\rangle$ be a formal series, we recall that the support of $S$ is given by:

$$
\operatorname{supp}(S)=\left\{v \in \Sigma_{\varepsilon}^{*}:\langle S, v\rangle \neq 0\right\} .
$$

We will call $(\mathbf{F F})$ the following condition:

(FF) For any $u \in \Sigma^{*}$, the set $\operatorname{supp}(S) \cap\left(\Phi^{-1}(u)\right)$ is finite.

If the formal series $S$ satisfies (FF), we say that it is $\Phi$-finite. The set of $\Phi$-finite series in $k\left\langle\left\langle\Sigma_{\varepsilon}\right\rangle\right\rangle$ is denoted $\left(k\left\langle\left\langle\Sigma_{\varepsilon}\right\rangle\right\rangle\right)_{\Phi-\text { finite }}$.

Theorem 4 The set $\left(k\left\langle\left\langle\Sigma_{\varepsilon}\right\rangle\right\rangle\right)_{\Phi-\text { fnite }}$ is closed under $+, \cdot, \alpha(?)$ and $(?) \alpha$.

Proof. $\operatorname{As} \operatorname{supp}\left(S_{1}+S_{2}\right) \subseteq \operatorname{supp}\left(S_{1}\right) \cup \operatorname{supp}\left(S_{2}\right), \operatorname{supp}\left(\alpha S_{1}\right) \subseteq \operatorname{supp}\left(S_{1}\right)$ and $\operatorname{supp}\left(S_{1} \alpha\right) \subseteq \operatorname{supp}\left(S_{1}\right)$ for $S_{1}, S_{2} \in k\left\langle\left\langle\Sigma_{\varepsilon}\right\rangle\right\rangle$ and $\alpha \in k$, the stability is shown for,$+ \alpha(?)$ and (?) $\alpha$.

Now, for the Cauchy product, one can check that :

$$
\operatorname{supp}\left(S_{1} S_{2}\right) \cap \Phi^{-1}(u) \subseteq \bigcup_{u=u_{1} u_{2}}\left(\operatorname{supp}\left(S_{1}\right) \cap \Phi^{-1}\left(u_{1}\right)\right)\left(\operatorname{supp}\left(S_{2}\right) \cap \Phi^{-1}\left(u_{2}\right)\right)
$$

which is a finite set if $S_{1}, S_{2} \in\left(k\left\langle\left\langle\Sigma_{\varepsilon}\right\rangle\right\rangle\right)_{\Phi \text {-finite }}$.

\section{Remark 4}

- Every polynomial is $\Phi$-finite.

- The star $S^{*}$ need not be $\Phi$-finite even if $S$ is $\Phi$-finite. The simplest example is provided by $S=\tilde{\varepsilon}$.

Next, we show that $\Phi: k\left\langle\Sigma_{\varepsilon}\right\rangle \mapsto k\langle\Sigma\rangle$ can be extended to $k\left\langle\left\langle\Sigma_{\varepsilon}\right\rangle\right\rangle_{\Phi \text {-finite }}$ as a polymorphism.

Theorem 5 For any $S, T \in\left(k\left\langle\left\langle\Sigma_{\varepsilon}\right\rangle\right\rangle\right)_{\Phi-f i n i t e}$,

$$
\begin{gathered}
\Phi(S+T)=\Phi(S)+\Phi(T), \Phi(S T)=\Phi(S) \Phi(T) \\
\Phi(\alpha S)=\alpha \Phi(S), \Phi(S \alpha)=\Phi(S) \alpha
\end{gathered}
$$

If $S^{*}$ is $\Phi$-finite, $\Phi\left(S^{*}\right)$ is a star of $\Phi(S)$. In particular, if $\Phi(S)$ has no constant term, one has

$$
\Phi\left(S^{*}\right)=(\Phi(S))^{*}
$$


Proof. For sum and Cauchy product, we obtain the result by the following relations:

$$
\begin{aligned}
& \sum_{v \in \Phi^{-1}(u)}\langle S+T, v\rangle=\sum_{v \in \Phi^{-1}(u)}\langle S, v\rangle \oplus \sum_{v \in \Phi^{-1}(u)}\langle T, v\rangle \\
& \sum_{v \in \Phi^{-1}(u)}\langle S T, v\rangle=\sum_{u=u_{1} u_{2}}\left(\sum_{v \in \Phi^{-1}\left(u_{1}\right)}\langle S, v\rangle \otimes \sum_{v \in \Phi^{-1}\left(u_{2}\right)}\langle T, v\rangle\right)
\end{aligned}
$$

Then $\Phi\left(S^{*}\right)$ is a solution of the equation $Y=\varepsilon+\Phi(S) Y$ as $S^{*}=\varepsilon+S S^{*}$, and then $\Phi\left(S^{*}\right)=\Phi(S)^{*}$.

A $\Phi$-finite series may be not rational.

Example(s) 4 The series in $\mathbb{N}\langle\langle\Sigma\rangle\rangle$

$$
S=\sum_{|u|_{a}=|u|_{\tilde{\varepsilon}}} u
$$

is not rational and however $\Phi$-finite.

We recall that a matrix $M \in k^{n \times n}$ is nilpotent if there exists a positive integer $N$ such that $M^{N}=0$.

Proposition 1 Let $S$ be a rational series in $k\left\langle\left\langle\Sigma_{\varepsilon}\right\rangle\right\rangle$ with $(\lambda, \mu, \gamma)$ a linear representation of $S$.

i) If $\mu$ is nilpotent then $S$ is $\Phi$-finite.

ii) Conversely, if $S$ is $\Phi$-finite, $k$ a field and $(\lambda, \mu, \gamma)$ is of minimal dimension then $\mu$ is nilpotent.

Proof. i) With the notations of the theorem, suppose that there is an integer $N$ such that $\mu(\tilde{\varepsilon})^{N}=0_{n \times n}$. Then, for $u=a_{1} a_{2} \cdots a_{k}$ one has

$$
\begin{array}{r}
\sum_{\Phi(v)=u}\langle S \mid v\rangle=\sum_{n_{0}, n_{1}, \cdots n_{k} \in \mathbb{N}}\left\langle S \mid \tilde{\varepsilon}^{n_{0}} a_{1} \tilde{\varepsilon}^{n_{1}} a_{2} \tilde{\varepsilon}^{n_{2}} \cdots a_{k} \tilde{\varepsilon}^{n_{k}}\right\rangle= \\
\sum_{n_{0}, n_{1}, \cdots n_{k} \in \mathbb{N}} \lambda \mu(\tilde{\varepsilon})^{n_{0}} \mu\left(a_{1}\right) \mu(\tilde{\varepsilon})^{n_{1}} \mu\left(a_{2}\right) \mu(\tilde{\varepsilon})^{n_{2}} \cdots \mu\left(a_{k}\right) \mu(\tilde{\varepsilon})^{n_{k}} \gamma= \\
\sum_{n_{0}, n_{1}, \cdots n_{k}<N} \lambda \mu(\tilde{\varepsilon})^{n_{0}} \mu\left(a_{1}\right) \mu(\tilde{\varepsilon})^{n_{1}} \mu\left(a_{2}\right) \mu(\tilde{\varepsilon})^{n_{2}} \cdots \mu\left(a_{k}\right) \mu(\tilde{\varepsilon})^{n_{k}} \gamma
\end{array}
$$

which is obviously finite.

ii) If $(\lambda, \mu, \gamma)$ is of minimal dimension $n$, then there exists words $\left(u_{i}\right)_{1 \leq i \leq n},\left(v_{j}\right)_{1 \leq j \leq n}$ in $\Sigma_{\varepsilon}$ such that the $n \times n$ matrices

$$
L=\left(\begin{array}{c}
\lambda \mu\left(u_{1}\right) \\
\lambda \mu\left(u_{2}\right) \\
\vdots \\
\lambda \mu\left(u_{n}\right)
\end{array}\right) \text { and } G=\left(\begin{array}{llll}
\mu\left(v_{1}\right) \gamma & \mu\left(v_{2}\right) \gamma & \cdots & \mu\left(v_{n}\right) \gamma
\end{array}\right)
$$


are regular ( $L$ is a block matrix of $n$ lines of size $1 \times n$ and $G$ is a block matrix of $n$ columns of size $n \times 1$; indeed, $L$ and $G$ are $n \times n$ square matrices.) [1].

Now, for $1 \leq i, j \leq n$ the family

$$
\left(\left\langle S \mid u_{i} \tilde{\varepsilon}^{n} v_{j}\right\rangle\right)_{n \geq 0}=\left(\lambda \mu\left(u_{i}\right) \mu\left(\tilde{\varepsilon}^{n}\right) \mu\left(v_{j}\right) \gamma\right)_{n \geq 0}
$$

as a subfamily of $(\langle S \mid v\rangle)_{\Phi(v)=\Phi\left(u_{i} v_{j}\right)}$ must be with finite support. This implies that $\left(L \mu\left(\tilde{\varepsilon}^{n}\right) G\right)_{n \geq 0}$ is with finite support. As $L$ and $G$ are invertible, $\mu(\tilde{\varepsilon})$ must be nilpotent.

\subsection{FC ( $\Phi$-convergent) series}

If we want to go further in the extension of $\Phi$ (and so doing to cover the - boolean - classical case), we must extend the domain of computability of the sums (10) to (some) countable families.

Many approaches exist in the literature [10], mainly by topology, ordered structure or "sum" function. Here, we adopt the last option with a minimal axiomatization adapted to our goal.

The semiring $k$ will be supposed endowed with a sum function $\mathfrak{s u m}$ taking some (at most) countable families $\left(a_{i}\right)_{i \in I}$ (called summable) and computing an element of $k$ denoted $\mathfrak{s u m}_{i \in I} a_{i}$. This function is subjected to the following axioms:

BCS1. - If $\left(a_{i}\right)_{i \in I}$ is finite, then it is summable and

$$
\mathfrak{s u m}_{i \in I} a_{i}=\sum_{i \in I} a_{i}
$$

CS2. - If $\left(a_{i}\right)_{i \in I}$ and $\left(b_{i}\right)_{i \in I}$ are summable, so is $\left(a_{i}+b_{i}\right)_{i \in I}$ and

$$
\mathfrak{s u m}_{i \in I} a_{i}+b_{i}=\left(\mathfrak{s u m}_{i \in I} a_{i}\right)+\left(\mathfrak{s u m}_{i \in I} b_{i}\right)
$$

CS3. - If $\left(a_{i}\right)_{i \in I}$ and $\left(b_{j}\right)_{j \in J}$ are summable, so is $\left(a_{i} b_{j}\right)_{(i, j) \in I \times J}$ and

$$
\mathfrak{s u m}_{(i, j) \in I \times J} a_{i} b_{j}=\left(\mathfrak{s u m}_{i \in I} a_{i}\right)\left(\mathfrak{s u m}_{j \in J} b_{j}\right)
$$

CS4. - If $\left(a_{i}\right)_{i \in I}$ is summable and $I=\sqcup_{\lambda \in \Lambda} J_{\lambda}$ is partitionned in finite subsets. Then $\left(\sum_{j \in J_{\lambda}} a_{j}\right)_{\lambda \in \Lambda}$ is summable and

$$
\mathfrak{s u m}_{i \in I} a_{i}=\mathfrak{s u m}_{\lambda \in \Lambda}\left(\sum_{j \in J_{\lambda}} a_{j}\right)
$$

CS5. - If $I=\sqcup_{\lambda \in \Lambda} J_{\lambda}$ with $\Lambda$ finite and each $\left(a_{j}\right)_{j \in J_{\lambda}}$ is summable. Then so is $\left(a_{i}\right)_{i \in I}$ and

$$
\mathfrak{s u m}_{i \in I} a_{i}=\sum_{\lambda \in \Lambda} \mathfrak{s u m}_{j \in J_{\lambda}} a_{j}
$$

CS6. - If $\left(a_{i}\right)_{i \in I}$ is summable and $\phi: J \mapsto I$ is one-to-one then $\left(a_{\phi(j)}\right)_{j \in J}$ is summable and

$$
\mathfrak{s u m}_{i \in I} a_{i}=\mathfrak{s u m}_{j \in J} a_{\phi(j)}
$$

Definition 2 A semiring with $\mathfrak{s u m}$ function (as above) which fulfills CS1..6 will be called a CS-semiring. 
If $k$ is a CS-semiring, the semiring of square matrices $k^{n \times n}$ will be equipped with the following $\mathfrak{s u m}$ function:

A family $\left(M^{(i)}\right)_{i \in I}$ of square matrices will be said summable iff it is so componentwise i.e. the $n^{2}$ families $\left(M_{r, s}^{(i)}\right)_{i \in I}$ (for $1 \leq r, s \leq n$ ) are summable. In this case, the sum of the family is the matrix $L$ such that, for $1 \leq r, s \leq n, L_{r s}=\mathfrak{s u m}_{i \in I} M_{r s}^{(i)}$ (i.e. the sum is computed componentwise). It can be easily checked that, with this sum function, $k^{n \times n}$ is a CS-semiring.

Remark 5 Let $k$ be a topological semiring (i.e. $k$ is endowed with some Hausdorff topology $\mathcal{T}$ such that the two binary operations - sum and product - are continuous mappings $k \times k \mapsto k$ ). We recall that a family $\left(a_{i}\right)_{i \in I}$ is said summable with sum $s$ iff it satisfies the following property, where $\mathfrak{B}(s)$ is a basis of neighbourhoods of $s$.

$$
(\forall V \in \mathfrak{B}(s))\left(\exists F \subset_{\text {finite }} I\right)\left(\forall F^{\prime}\right)\left(F \subset F^{\prime} \subset_{\text {finite }} I \Longrightarrow \sum_{i \in F^{\prime}} a_{i} \in V\right) .
$$

In this case the axioms $\mathbf{C S 1 2 4 5 6}$ are automatically satisfied for the preceding (usual) notion of summability.

Example(s) 5 Below some examples of CS-semirings which are metric semirings (i.e. the notion of summability and the sum function are given as in Remark (5)).

1. The fields $\mathbb{Q}, \mathbb{R}, \mathbb{C}$ with their usual metric.

2. Any semiring with the discrete topology, given by the metric $d(x, y)=1$ if $x \neq y$ and $d(x, x)=0$.

3. The extended integers $(\mathbb{N} \cup\{+\infty\},+, \times)$ with the Frechet topology given by the metric $d(n, m)=$ $\left|\frac{1}{n}-\frac{1}{m}\right|$ and $d(+\infty, n)=\frac{1}{n}$.

4. The (min,plus) closed half-ray $\left([0,+\infty]_{\overline{\mathbb{R}}}\right.$, min,+$)$ with the metric transported by the rational homomorphism $x \mapsto \frac{x}{x+1}$ from $[0,+\infty]_{\mathbb{R}}$ to $[0,1]_{\mathbb{R}}$ i.e. with $d(x, y)=\left|\frac{x}{x+1}-\frac{y}{y+1}\right|$ and with $\left.\frac{x}{x+1}\right|_{x=+\infty}=1$.

Let $S \in k\left\langle\left\langle\Sigma_{\varepsilon}\right\rangle\right\rangle$ be a formal series, we will call (FC) the following condition:

(FC) For any $u \in \Sigma^{*}$, the (countable) family $(\langle S \mid v\rangle)_{v \in \Phi^{-1}(u)}$ is summable.

If the formal series $S$ satisfies (FC), we say that it is $\Phi$-convergent. The set of $\Phi$-convergent series in $k\left\langle\left\langle\Sigma_{\varepsilon}\right\rangle\right\rangle$ is denoted $k\left\langle\left\langle\Sigma_{\varepsilon}\right\rangle\right\rangle_{\Phi-\text { conv }}$.

It is straightforward that a $\Phi$-finite series is $\Phi$-convergent. We have now a theorem similar to Theorem (4) for $k\left\langle\left\langle\Sigma_{\varepsilon}\right\rangle\right\rangle_{\Phi-\text { conv }}$.

Theorem 6 The set $k\left\langle\left\langle\Sigma_{\varepsilon}\right\rangle\right\rangle_{\Phi-c o n v}$ is closed under $+, \cdot, \alpha(?)$ and $(?) \alpha$.

Proof. Stability by,$+ \alpha(?)$ and (?) $\alpha$ is straightforward using the axioms CS123. Let us give the details of the proof for the Cauchy product. We have to prove that, for every $S, T \in k\left\langle\left\langle\Sigma_{\varepsilon}\right\rangle\right\rangle_{\Phi \text {-conv }}$ and $u \in \Sigma^{*}$, the (countable) family

$$
(\langle S T \mid v\rangle)_{v \in \Phi^{-1}(u)}=(\langle S T \mid v\rangle)_{\Phi(v)=u}
$$


is summable.

From the definition of the Cauchy product we have the finite sums

$$
\langle S T \mid v\rangle=\sum_{v_{1} v_{2}=v}\left\langle S \mid v_{1}\right\rangle\left\langle T \mid v_{2}\right\rangle
$$

and, from CS4, the summability would be a consequence of that of the family

$$
\left(\left\langle S \mid v_{1}\right\rangle\left\langle T \mid v_{2}\right\rangle\right)_{\substack{\begin{subarray}{c}{(v)=u \\
v=v_{1} v_{2}} }} \\
{v}\end{subarray}}=\left(\left\langle S \mid v_{1}\right\rangle\left\langle T \mid v_{2}\right\rangle\right)_{\Phi\left(v_{1} v_{2}=u\right)}
$$

(with the same sum). This family can be partitionned in a finite sum of families (with the same sum)

$$
\sqcup_{u_{1} u_{2}=u}\left(\left\langle S \mid v_{1}\right\rangle\left\langle T \mid v_{2}\right\rangle\right)_{\substack{v_{1} \in \Phi^{-1}\left(u_{1}\right) \\ v_{2} \in \Phi^{-1}\left(u_{2}\right)}}
$$

each of which, by CS3, is summable. Thus, by CS5, the family (23) is summable and hence summability of (22) (with the same sum) follows.

Next, we show that $\Phi:\left(k\left\langle\left\langle\Sigma_{\varepsilon}\right\rangle\right\rangle\right)_{\Phi-\text { conv }} \rightarrow k\langle\langle\Sigma\rangle\rangle$ is a polymorphism.

Theorem 7 For any $S, T \in\left(k\left\langle\left\langle\Sigma_{\varepsilon}\right\rangle\right\rangle\right)_{\Phi-c o n v}$,

$$
\begin{gathered}
\Phi(S+T)=\Phi(S)+\Phi(T), \Phi(S T)=\Phi(S) \Phi(T) \\
\Phi(\alpha S)=\alpha \Phi(S), \Phi(S \alpha)=\Phi(S) \alpha
\end{gathered}
$$

If $S^{*}$ is $\Phi$-conv, $\Phi\left(S^{*}\right)$ is a star of $\Phi(S)$. In particular, if $\Phi(S)$ has no constant term, one has

$$
\Phi\left(S^{*}\right)=(\Phi(S))^{*}
$$

Proof. The proof is similar to that of Theorem (5), using the axioms of CS-semirings.

In the sequel, as in the classical case, the summability of $\left(\mu(\tilde{\varepsilon})^{n}\right)_{n \in \mathbb{N}}$ will play a central rôle. We will then call closable a square matrix $M \in k^{n \times n}$ such that the family $\left(M^{n}\right)_{n \in \mathbb{N}}$ is summable. Note that, in this case, the sum $\mathfrak{s u m}_{n \in \mathbb{N}} M^{n}$ is a two-sided (we could say "topological") star of $M$.

For example, with the boolean semiring endowed with the discrete topology, every $M \in \mathbb{B}^{n \times n}$ is closable (i.e. the sequence $S_{N}=\sum_{k=0}^{N} M^{k}$ is stationnary).

We have the following theorem, very similar to (1).

Proposition 2 Let $S$ be a rational series in $k\left\langle\left\langle\Sigma_{\varepsilon}\right\rangle\right\rangle$ ( $k$ a CS semiring) with $(\lambda, \mu, \gamma)$ a linear representation of $S$.

(i) If $\mu(\tilde{\varepsilon})$ is closable then $S$ is $\Phi$-convergent.

(ii) Conversely, if $S$ is $\Phi$-convergent, $k=\mathbb{R}, \mathbb{C}$ and $(\lambda, \mu, \gamma)$ minimal then $\mu(\tilde{\varepsilon})$ is closable.

Proof. The proof (i) is similar to that of Theorem (1). The first computation of (ii) is similar, but, to conclude, we use the property (which holds in $\mathbb{R}$ and $\mathbb{C}$ ) that a family is summable iff it is absolutely summable (because of CS6) and then subfamilies of summable families are summable. 


\section{Equivalence}

We now deal with an algebraic method to eliminate the $\varepsilon$-transitions from a weighted $\varepsilon$-automaton $\mathcal{A}_{\varepsilon}$. The result is a weighted automaton with behaviour $\Phi\left(\right.$ behaviour $\left.\left(\mathcal{A}_{\varepsilon}\right)\right)$.

Theorem 8 Let $k$ be a CS semiring and $\mathcal{A}_{\varepsilon}=(\lambda, \mu, \gamma)$ be a weighted $\varepsilon$-automaton with weights in $k$. We suppose that $\mu(\tilde{\varepsilon})$ is closable. Then

i) the series behaviour $\left(\mathcal{A}_{\varepsilon}\right)$ is $\Phi$-convergent.

ii) there exists a (algorithmically constructible) weighted automaton $\mathcal{A}=\left(\lambda^{\prime}, \mu^{\prime}, \gamma^{\prime}\right)$ such that

$$
\operatorname{behaviour}(\mathcal{A})=\Phi\left(\text { behaviour }\left(\mathcal{A}_{\varepsilon}\right)\right) \text {. }
$$

Proof. The point i) is a reformulation of Proposition (2). Remark that, if $\left(\mu(\tilde{\varepsilon})^{n}\right)_{n \in \mathbb{N}}$ is summable its sum is a (two sided) star of $\mu(\tilde{\varepsilon})$ that, for convenience, we will denote $\mu(\tilde{\varepsilon})^{*}$.

Let $B$ be the behaviour of $\mathcal{A}_{\varepsilon}$ one has

$$
\Phi(B)=\sum_{u \in \Sigma^{*}}\left(\sum_{\Phi(v)=u}\langle B \mid v\rangle\right) u=\sum_{u \in \Sigma^{*}}\left(\sum_{\Phi(v)=u} \lambda \mu(v) \gamma\right) u
$$

Let, now $u=a_{1} a_{2} \cdots a_{n} \in \Sigma^{*}$, one has

$$
\begin{array}{r}
\sum_{\Phi(v)=u} \lambda \mu(v) \gamma=\lambda\left(\sum_{k_{0}, k_{1}, \cdots k_{n} \in \mathbb{N}} \mu(\tilde{\varepsilon})^{k_{0}} \mu\left(a_{1}\right) \mu(\tilde{\varepsilon})^{k_{1}} \cdots \mu\left(a_{n}\right) \mu(\tilde{\varepsilon})^{k_{n}}\right) \gamma= \\
\lambda \mu(\tilde{\varepsilon})^{*} \mu\left(a_{1}\right) \mu(\tilde{\varepsilon})^{*} \cdots \mu\left(a_{n}\right) \mu(\tilde{\varepsilon})^{*} \gamma=\lambda\left(\mu(\tilde{\varepsilon})^{*} \mu\left(a_{1}\right)\right)\left(\mu(\tilde{\varepsilon})^{*} \mu\left(a_{2}\right)\right) \cdots\left(\mu(\tilde{\varepsilon})^{*} \mu\left(a_{n}\right)\right)\left(\mu(\tilde{\varepsilon})^{*} \gamma\right)
\end{array}
$$

the conclusion follows taking, for all $a \in \Sigma$,

$$
\left(\lambda^{\prime}, \mu^{\prime}(a), \gamma^{\prime}\right)=\left(\lambda, \mu(\tilde{\varepsilon})^{*} \mu(a), \mu(\tilde{\varepsilon})^{*} \gamma\right) .
$$

Let $k$ be a semiring and $\mathcal{A}_{\varepsilon}=(\lambda, \mu, \gamma)$ a $k$ - $\varepsilon$-automaton with $\left.S \in k\left\langle\left\langle\Sigma_{\varepsilon}\right\rangle\right\rangle\right)_{\Phi \text {-conv }}$ as behaviour. Theorem (2) gives the lower bounds if the set of coefficients is the semiring $k$ (resp. ring, field).

Proposition 3 We suppose that, in $k^{n \times n}$, stars are unique. Let $(\lambda, \mu, \gamma)$ a $k$ - $\varepsilon$-automaton such that $\mu(\tilde{\varepsilon})$ admits a tree of computation and is closable, then the complexity of the elimination of $\varepsilon$-transitions which produces the weighted automaton $\mathcal{A}=\left(\lambda^{\prime}, \mu^{\prime}, \gamma^{\prime}\right)$, is $O\left(n^{\omega}\right)$.

Proof. The unique matrix $\mu_{\tilde{\varepsilon}}^{*}$ given by the algorithm of Theorem (1) satisfies the relation:

$$
\mu_{\tilde{\varepsilon}}^{*}=\mu_{\tilde{\varepsilon}}^{*} \mu_{\tilde{\varepsilon}}^{m+1}+\mu_{\tilde{\varepsilon}}^{m}+\cdots+\mu_{\tilde{\varepsilon}}+1_{n \times n} .
$$

for all $m>0$. In fact, by induction,

$$
\mu^{*} \mu^{m+2}+\mu^{m+1}+\cdots+\mu+1=\left(\mu^{*} \mu^{m+1}+\mu^{m}+\cdots+1\right) \mu+1=\mu^{*} \mu+1=\mu^{*} .
$$

As $\left(\mu^{m}\right)_{m \in \mathbb{N}}$ is summable, one has $\mu^{*}=\sum \mu^{m}$. Next set $\lambda^{\prime}=\lambda, \gamma^{\prime}=\mu_{\tilde{\varepsilon}}^{*} \gamma$ and $\mu^{\prime}(a)=\mu_{\tilde{\varepsilon}}^{*} \mu(a)$ for each letter $a \in \Sigma$. 


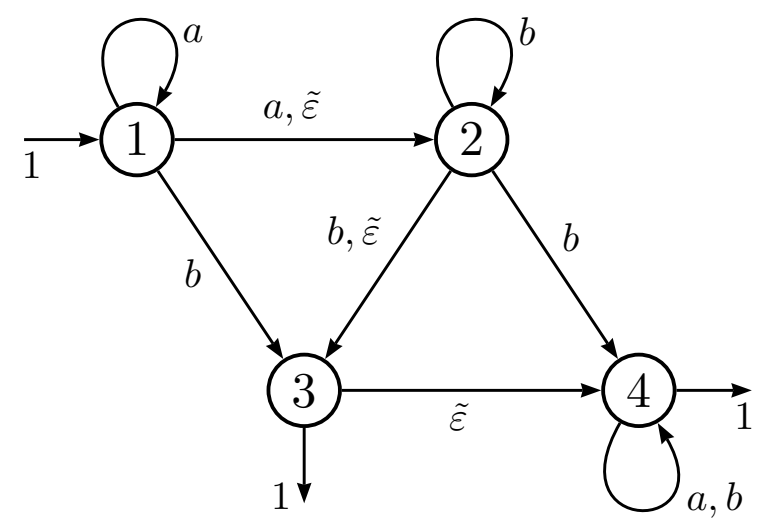

Figure 3: $A \mathbb{B}-\varepsilon$-automaton

Remark 6 One could also with the same result set $\lambda^{\prime}=\lambda \mu_{\tilde{\varepsilon}}^{*}, \mu^{\prime}(a)=\mu(a) \mu_{\tilde{\varepsilon}}^{*}$ for each letter $a \in \Sigma$ and $\gamma^{\prime}=\gamma$.

In the following, we have an example of a boolean automaton with $\varepsilon$-transitions.

Example(s) 6 The linear representation of Figure 3 is:

$\lambda=\left(\begin{array}{llll}1 & 0 & 0 & 0\end{array}\right), \mu_{\tilde{\varepsilon}}=\left(\begin{array}{cccc}0 & 1 & 0 & 0 \\ 0 & 0 & 1 & 0 \\ 0 & 0 & 0 & 1 \\ 0 & 0 & 0 & 0\end{array}\right), \mu(a)=\left(\begin{array}{cccc}1 & 1 & 0 & 0 \\ 0 & 0 & 0 & 0 \\ 0 & 0 & 0 & 0 \\ 0 & 0 & 0 & 1\end{array}\right), \mu(b)=\left(\begin{array}{cccc}0 & 0 & 1 & 0 \\ 0 & 1 & 1 & 1 \\ 0 & 0 & 0 & 0 \\ 0 & 0 & 0 & 1\end{array}\right)$ and $\gamma=\left(\begin{array}{l}0 \\ 0 \\ 1 \\ 1\end{array}\right)$

By computation:

$$
\begin{aligned}
& \mu_{\tilde{\varepsilon}}^{*}=\left(\begin{array}{llll}
1 & 1 & 1 & 1 \\
0 & 1 & 1 & 1 \\
0 & 0 & 1 & 0 \\
0 & 0 & 0 & 1
\end{array}\right), \lambda^{\prime}=\left(\begin{array}{llll}
1 & 0 & 0 & 0
\end{array}\right), \mu^{\prime}(a)=\mu_{\tilde{\varepsilon}}^{*} \mu(a)=\left(\begin{array}{cccc}
1 & 1 & 0 & 1 \\
0 & 0 & 0 & 1 \\
0 & 0 & 0 & 0 \\
0 & 0 & 0 & 1
\end{array}\right), \\
& \mu^{\prime}(b)=\mu_{\tilde{\varepsilon}}^{*} \mu(b)=\left(\begin{array}{llll}
0 & 1 & 1 & 1 \\
0 & 1 & 1 & 1 \\
0 & 0 & 0 & 0 \\
0 & 0 & 0 & 1
\end{array}\right) \text { and } \gamma^{\prime}=\mu_{\tilde{\varepsilon}}^{*} \gamma=\left(\begin{array}{l}
1 \\
1 \\
1 \\
1
\end{array}\right),
\end{aligned}
$$

The resulting boolean automaton is presented in Figure 4 and its linear representation is $\left(\lambda^{\prime}, \mu^{\prime}, \gamma^{\prime}\right)$. 


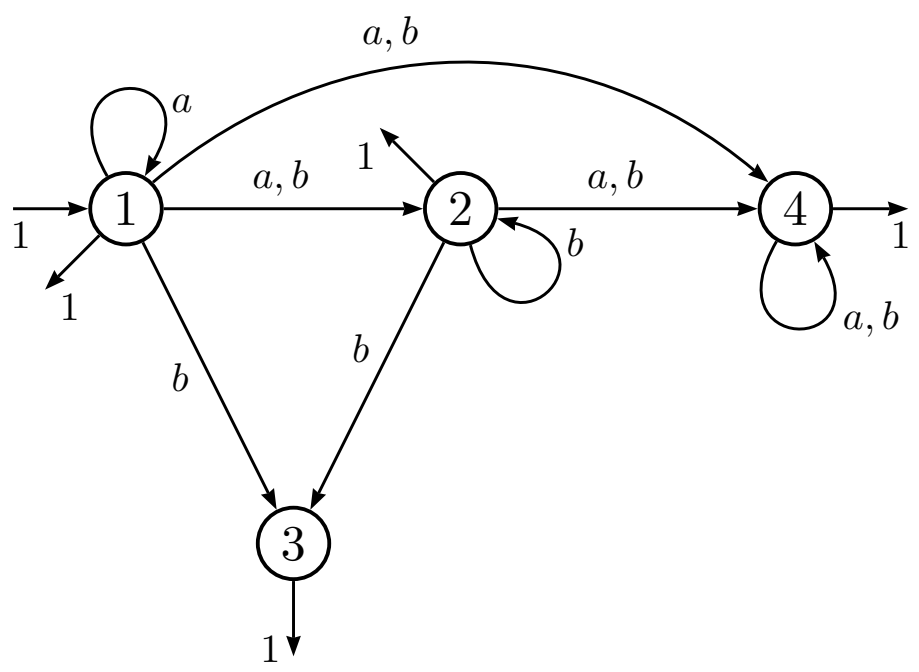

Figure 4: $\mathrm{A} \mathbb{B}$-automaton

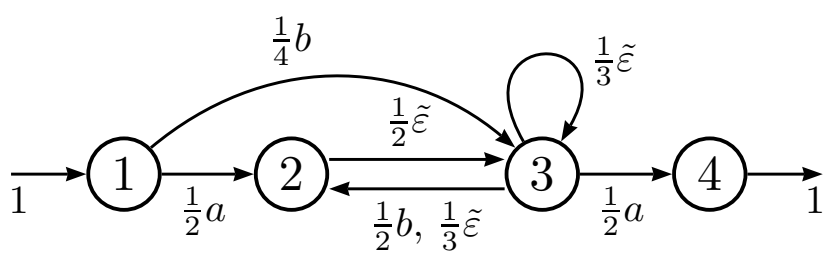

Figure 5: $\mathrm{A} \mathbb{Q}-\varepsilon$-automaton 


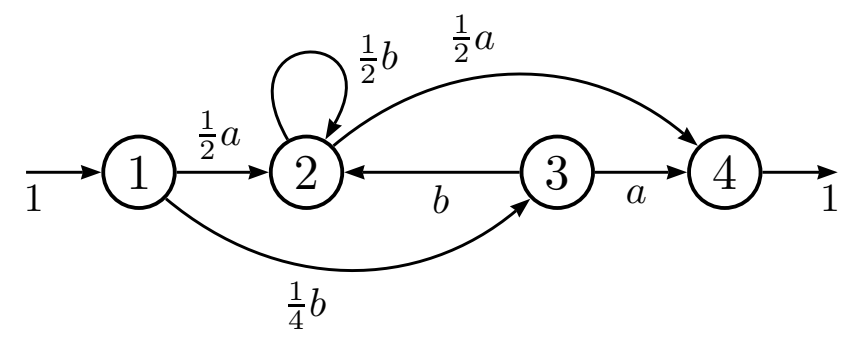

Figure 6: $A \mathbb{Q}$-automaton

In the next example, our algebraic method is applied on a $\mathbb{Q}-\varepsilon$-automaton.

Example(s) 7 The linear representation of Figure 5 is:

$\lambda=\left(\begin{array}{llll}1 & 0 & 0 & 0\end{array}\right), \mu_{\tilde{\varepsilon}}=\left(\begin{array}{cccc}0 & 0 & 0 & 0 \\ 0 & 0 & \frac{1}{2} & 0 \\ 0 & \frac{1}{3} & \frac{1}{3} & 0 \\ 0 & 0 & 0 & 0\end{array}\right), \mu(a)=\left(\begin{array}{cccc}0 & \frac{1}{2} & 0 & 0 \\ 0 & 0 & 0 & 0 \\ 0 & 0 & 0 & \frac{1}{2} \\ 0 & 0 & 0 & 0\end{array}\right), \mu(b)=\left(\begin{array}{cccc}0 & 0 & \frac{1}{4} & 0 \\ 0 & 0 & 0 & 0 \\ 0 & \frac{1}{2} & 0 & 0 \\ 0 & 0 & 0 & 0\end{array}\right)$, and $\gamma=\left(\begin{array}{l}0 \\ 0 \\ 0 \\ 1\end{array}\right)$

By computation:

$\mu_{\tilde{\varepsilon}}^{*}=\left(\begin{array}{cccc}1 & 0 & 0 & 0 \\ 0 & \frac{4}{3} & 1 & 0 \\ 0 & \frac{2}{3} & 2 & 0 \\ 0 & 0 & 0 & 1\end{array}\right), \lambda^{\prime}=\left(\begin{array}{cccc}1 & 0 & 0 & 0\end{array}\right), \mu^{\prime}(a)=\mu_{\tilde{\varepsilon}}^{*} \mu(a)=\left(\begin{array}{cccc}0 & \frac{1}{2} & 0 & 0 \\ 0 & 0 & 0 & \frac{1}{2} \\ 0 & 0 & 0 & 1 \\ 0 & 0 & 0 & 0\end{array}\right)$,

$\mu^{\prime}(b)=\mu_{\tilde{\varepsilon}}^{*} \mu(b)=\left(\begin{array}{cccc}0 & 0 & \frac{1}{4} & 0 \\ 0 & \frac{1}{2} & 0 & 0 \\ 0 & 1 & 0 & 0 \\ 0 & 0 & 0 & 0\end{array}\right)$ and $\gamma^{\prime}=\mu_{\tilde{\varepsilon}}^{*} \gamma=\left(\begin{array}{c}0 \\ 0 \\ 0 \\ 1\end{array}\right)$.

The resulting automaton is presented in Figure 6 and its linear representation is $\left(\lambda^{\prime}, \mu^{\prime}, \gamma^{\prime}\right)$.

\section{Conclusion}

Algebraic elimination for $\varepsilon$-automata has been presented. The problem of removing the $\varepsilon$-transitions is originated from generic $\varepsilon$-removal algorithm for weighted automata [15] using Floyd-Warshall and generic single-source shortest distance algorithms. Here, we have the same objective but the methods and algorithms are different. In [15], the principal characteristics of semirings used by the algorithm as well as the complexity of different algorithms used for each step of the elimination are detailed. The case of acyclic and non acyclic automata are analysed differently. Our algorithm here works with any semiring (supposing only that $\mu(\tilde{\varepsilon})$ is closable and with unique star) and the complexity is unique for the case of 
acyclic or non acyclic automata. This algorithm is even more efficient when the considered semiring is a ring.

\section{References}

[1] Berstel J., Reutenauer C., "Rational Series and Their Languages". EATCS, Monographs on Theoretical Computer Science, Springer Verlag, Berlin (1988).

[2] Champarnaud J.-M., Duchamp G., "Brzozowski's derivatives extended to multiplicities". Lectures Notes in Computer Science 2494 (2001), 52-64.

[3] Coppersmith D., Winograd S., "Matrix Multiplication via arithmetic progressions". Journal of Symbolic Computation 9 (1990), 251-280.

[4] Conway J.H., "Regular Algebra and Finite Machines". Chapman and Hall (1971).

[5] Duchamp G., Flouret M., Laugerotte É., Luque J-G., "Direct and dual laws for automata with multiplicities". Theoretical Computer Science 267 (2000) 105-120.

[6] Duchamp G., Reutenauer C., "Un critère de rationalité provenant de la géométrie non- commutative". Inventiones Mathematicae 128 (1997).

[7] Hebisch U., Weinert H. J., "Semirings - Algebraic Theory and Applications in computer Science". Word Scientific Publishing, Singapore (1993).

[8] Heyting A., "Die Theorie der Linearen Gleichungen in einer Zahlenspezies mit nichtkommutativer Multiplikation". Math. Ann. 98 (1927) 465-490.

[9] Huss-Lederman S., Jacobson E.M., Johnson J.R., Tsao A., Turnbull T., "Implementation of Strassen's Algorithm for Matrix Multiplication". Proceeding of the ACM/IEEE conference on supercomputing, Pittsburgh, Pennsylvania, USA (1996).

[10] Kuich W., Salomaa A., "Semirings, Automata, Languages". EATCS, Monographs on Theoretical Computer Science Volume 5, Springer Verlag, Berlin (1986).

[11] Krob D., "The equality problem for rational series with multiplicities in the tropical semiring is undecidable". International Journal of Algebra and Computation, 4(3) (1994) 405-425.

[12] Krob D., "Some automata-theoretic aspects of Min-Max-Plus semirings". Chapter in Idempotency Analysis. Number 11 in Publications of the Newton Institute (1998).

[13] Krob D., “ Algorithms, automata, complexity and games”. Theoretical Computer Science 89(2) (1991) 207-345.

[14] Laugerotte É., Ziadi D., "Weighted recognition”. Journal of Automata, Langugages and combinatorics, (in preparation). 
[15] Mohri M., "Generic $\varepsilon$-Removal Algorithm for Weighted Automata". Lecture Notes in Computer Science volume 2088 (2001) 230-242, Springer Verlag, Berlin, (2001).

[16] Richardson A. R., "Simultaneous Linear equations over a division ring". Proc. Lond. Math. Soc. 28 (1928) 395-420.

[17] Schützenberger, "On the definition of a family of automata". Inform. and Contr. 4 (1961) 245-270.

[18] Schönhage A., Strassen V., "Schnelle Multiplikation grosser Zahlen”. Computing 7 (1971) 281292.

[19] Strassen V., "Gaussian Elimination is not optimal". Numerische Mathematik 13 (1969) 354-356. 
